${ }^{1}$ Molecular Oncology and Genetics Department, Innovative Medical Forum, The F. Lukaszczyk Oncology Center Bydgoszcz, Poland 2Department of Oncology, Radiotherapy and Gynecologic Oncology, Collegium Medicum, Nicolaus Copernicus University, Bydgoszcz, Poland

${ }^{3}$ Department of Thoracic Surgery and Tumors, Faculty of Medicine, Collegium Medicum, Nicolaus Copernicus University, Bydgoszcz, Poland

\title{
A preliminary study on MTDH expression as a potential prognostic cancer marker
}

\author{
Corresponding author: \\ Krzysztof Roszkowski \\ Department of Oncology, Radio- \\ therapy and Gynecologic Oncology, \\ Collegium Medicum, Nicolaus Coper- \\ nicus University, Bydgoszcz 85-796, \\ Romanowskiej 2; Poland \\ tel. +48523743744 \\ e-mail: roszkowskik@cm.umk.pl
}

Medical Research Journal 2018; Volume 3, Number 3, 211-214 10.5603/MRJ.a2018.0036 Copyright (C) 2018 Via Medica ISSN 2451-2591

\begin{abstract}
Background: Clinical studies have revealed that MTDH is overexpressed in various malignancies and is associated with disease progression and poor clinical outcomes. In order to study MTDH prognostic potential, we decided to evaluate MTDH expression changes using cancerous and non-cancerous cells lines. Secondly, for the first time, we evaluated MTDH expression in prostate cancer cell lines representing different metastatic potential in vivo

Methods: MTDH and PBGD (control) genes expression were measured by reverse transcription-quantitative polymerase chain reaction assay using Universal Probe Library in cancerous and non-cancerous cell lines. Results: MTDH gene expression analysis showed a decrease in colorectal cancer cell lines (Caco2, HT29) compared to non-cancerous cells lines (VH10, VH25, Hek293). The mean level of the MTDH mRNA expression, normalized in relation to the reference gene PBGD, increased in the prostate cancer cell lines as follows: PC3 (0.62 \pm 0.07$)$, PC3M (1.02 \pm 0.17$)$, PC3MPro4 (1.20 \pm 0.22$)$, and reached the highest value in PC3M4 (1.86 \pm 0.48$)$. In VH10, the expression of this gene was at $1.0 \pm 0.07$.

Conclusions: Our MTDH gene expression data are consistent with Mtdh protein expression analyzed in The Human Protein Atlas (HPA). Increasing MTDH expression is a promising prognostic factor. Key words: MTDH (Metadherin), prostate cancer cell lines, gene expression
\end{abstract}

Med Res J 2018; 3 (4): 211-214

\section{Introduction}

Bioinformatics analysis of pharmacogenetic $(60$ cell lines, including 58 cancer lines, with a specific cytogenetic profile and the gene expression profile, tested for the resistance or sensitivity to the 24,000 new chemicals) identified the $8 \mathrm{q} 22$ region as potentially associated with response to chemotherapy [1]. Studies using high-throughput technologies allowed the characterization of the high correlation between the amplification of genes on chromosome 8q22 and early recurrence of cancer [2-4].

There are 13 known genes in that region of chromosome, including MTDH which plays a key role in the metastasis [4]. Interestingly, MTDH has 30-40\% higher expression in breast cancer what accelerates the formation of metastasis and increases chemoresistance [4]. The MTDH gene was first identified in PHFA cells (Primary Human Fetal Astrocytes) after inducing with HIV-1 (Human Immunodeficiency Virus-1) or exposure to HIV-1 envelope glycoprotein [5, 6].
MTDH (Metadherin) firstly called AEG-1 (Astrocyte Elevated Gene-1), then LYRIC (3D3 and LYsine-RIch CEACAM1 co-isolated) is a gene that encodes a protein involved in physiological processes, such as regulating differentiation and proliferation of progenitor cells at early stages of embryonic development as part of the RNA-induced silencing complex (RISC) that participates in gene silencing. However, the protein is also involved in some pathological conditions, including cancer metastasis, increased proliferation and multidrug resistance [7-9].

Recently, clinical studies have revealed that MTDH is overexpressed in various cancers and associated with disease progression and poor clinical outcomes [10]. Moreover, MTDH has been found to promote cancer metastasis, chemoresistance, invasion, angiogenesis [7-9] and a key player in radioresistance regulation [11].

In this study, we analyzed the expression of the MTDH gene in the cancerous and noncancerous cells. Moreover, we decided to check how the expression of MTDH changes with the advancement of 
prostate cancer. For this purpose, we used a research model of cell lines derived from the PC3 line: PC3M, PC3MLN4 and PC3MPro4 ref: Pettaway CA, Pathak S, 1996 https://www.ncbi.nlm.nih.gov/pubmed/9816342), which present various degrees of advancement of the same cancer.

\section{Methods}

\section{Cell line cultures}

The PC3, PC3M, PC3MLN4 and PC3MPro4 cell lines [12] were cultured in the RPMI medium, while the $\mathrm{VH} 10$, CACO2, HT29 and Hek293 cell lines were cultured in the DMEM medium (both from BioWest SAS (France) with $10 \%$ fetal bovine serum (FBS) (Pan-Biotech $\mathrm{GmbH}$, Germany) and a 5\% antibiotic and antimycotic solution (BioWest SAS) at $37^{\circ} \mathrm{C}$ in a $5 \% \mathrm{CO} 2$ atmosphere at a constant humidity.

\section{mRNA isolation}

mRNA isolation was performed using the High Pure RNA Isolation Kit from Roche Diagnostics $\mathrm{GmbH}$ (Germany) according to the manufacturer's protocol. The method includes lysis of cells and subsequent separation of RNA on silica columns. RNA from each cell line was isolated in triplicate.

\section{Reverse transcription reaction}

cDNA synthesis was carried out using the Transcriptor High Fidelity kit cDNA Synthesis Kit from Roche Diagnostics $\mathrm{GmbH}$ according to the manufacturer's protocol, using two types of primers: Anchored oligo (dT) 18 Primer and Random Hexamer Primer.

\section{Real-Time PCR}

Real-time PCR was carried out using primers with the following sequences: reverse primer 5'-gaaactggctcagcagtagacc -3'; forward primer 5'- actggagatgctaatacaaatgga -3 ', and the TaqMan UPL 60 probe. The primers were selected using the free Universal Probe Library Assay Design Center application available online at www.universalprobelibrary.com. The porphobilinogen deaminase (PBGD) gene (Universal Probe Library Human PBGD Gene Assay, Roche, Life Science) was used as a reference gene for the normalization of the obtained data. The real-time PCR reaction was performed using Light Cycler 2.0 PCR System Roche Diagnostics $\mathrm{GmbH}$ (Germany) in the following conditions: one cycle at $95^{\circ} \mathrm{C} / 10 \mathrm{~min} ; 45$ cycles of denaturation $\left(95^{\circ} \mathrm{C} / 10 \mathrm{sec}\right)$, annealing $\left(60^{\circ} \mathrm{C} / 30 \mathrm{sec}\right)$ and extension $\left(72^{\circ} \mathrm{C} / 1 \mathrm{sec}\right)$ with data collection in two channels: $530 \mathrm{~nm}$ for FAM and

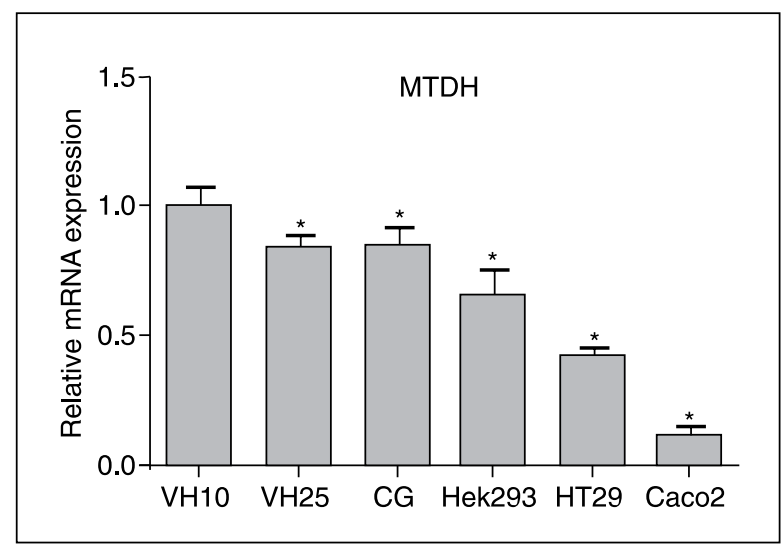

Figure 1. Results of the MTDH gene expression. Analyses were performed three times in three biological replicates (9 repetitions in total) and results were normalized with PBGD as the reference gene. The graph presents cell lines showing a decrease in the MTDH gene expression in colorectal cancer cell lines compared to non-cancerous cells lines (VH10, VH25, Hek293)

$610 \mathrm{~nm}$ for Light Cycler $^{\circledR}$ Yellow 555. Relative mRNA expression was calculated using the $2^{-\Delta \Delta C t}$ method.

\section{Results}

MTDH gene expression was measured in cancerous (Caco2, HT29, PC3, PC3M, PC3MLN4, PC3MPro) and non-cancerous cell lines (HEK293 and fibroblasts: $\mathrm{VH} 10$ and $\mathrm{VH} 25)$ using quantitative qPCR. All results were performed three times in three biological replicates and normalized with PBGD expression. We observed a statistically significant decrease of MTDH expression in Caco2, HT29 and PC3 cells in comparison to fibroblasts. Furthermore, our analysis conducted in the PC3, Caco2 and non-cancerous cell lines HEK293 cell demonstrated levels of the MTDH gene expression similar to those reported by the HPA (Human Protein Atlas) project available online.

The mean level of the MTDH mRNA expression, normalized in relation to the reference gene $P B G D$, increased in the prostate cancer cell lines as follows: PC3 (0.62 \pm 0.07$)$, PC3M (1.02 \pm 0.17$)$, PC3MPro4 (1.20 \pm 0.22$)$, and reached the highest value in PC3M4 (1.86 \pm 0.48$)$. In VH10, the expression of this gene was at $1.0 \pm 0.07$ (Fig. 2).

\section{Discussion}

Overexpression of MTDH gene was noticed in multiple cancers including colorectal cancer [13] breast cancer [14], prostate cancer [15] and glioma [16]. 


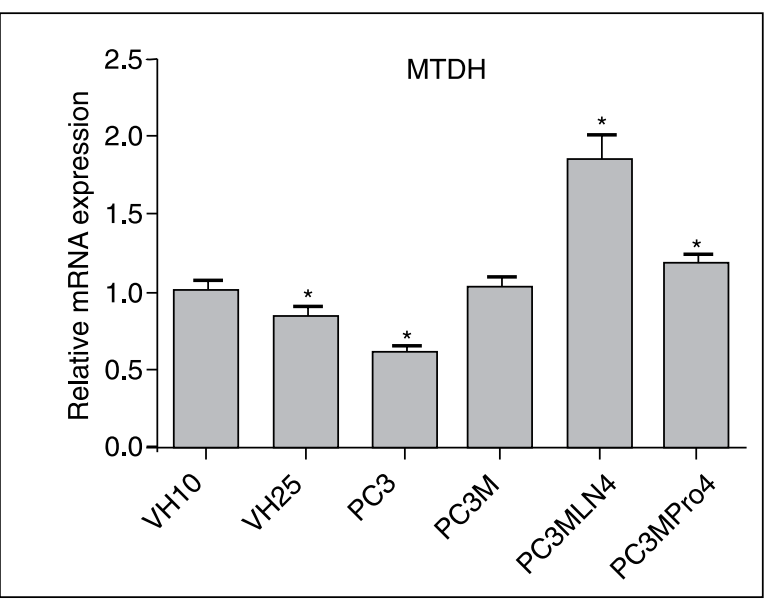

Figure 2. Relative levels of the MTDH gene expression in prostate cancer cell lines and fibroblasts. The level of expression was normalized in relation to the reference gene $P B G D$, with values standardized in relation to $\mathrm{VH} 10$. The graph shows mean values from 9 analysis; \pm SD. Asterisks indicate statistically significant values $(p \leq 0.05)$ compared to $\mathrm{VH} 10$. The graph presents cell lines showing an increase in the MTDH gene expression in PC3MLN4, PC3MPro compared to $\mathrm{VH} 10$ fibroblasts.

The expression of this gene was also analyzed as part of the large-scale project - The Human Protein Atlas (HPA) [17] at the protein as well as mRNA level in various tissues and cell lines. In our study, we compared MTDH mRNA expression between cancerous (CG, HT29, Caco2, PC3, PC3M, PC3MLN4, PC3Mpro4) and noncancerous cell lines (VH10, VH25, Hek293). Some of these cell lines were included in HPA, which gave us the opportunity to assess the compliance of our results. HPA included six different fibroblast cell lines, of which five were foreskin. We also chose two human foreskin fibroblasts: $\mathrm{VH} 10$ and $\mathrm{VH} 25$ as wild-type reference cell lines. Another noncancerous cell line, Hek293, was underexpressed compared to fibroblasts in HPA as well as in our analysis.

Our results of MTDH expression pattern in cancerous cell lines were also consistent with the previous results. Caco2 was one of the cell lines analyzed in HPA with the lowest expression of MTDH gene. We also noticed the lowest MTDH expression in Caco2. On the other hand, PC3 cell line showed a slightly decrease in MTDH expression in this study as well as in HPA

The consistency of the results obtained in this work with the previous research encouraged us to study other cell lines: CG and HT29. HT29 is another cell line derived from colorectal adenocarcinoma and, like Caco2, showed statistically significant lower expression level of MTDH compared to VH10. Moreover, we analyzed MTDH expression in CG cell line. It was between VH10 and Hek293, with was consistent with results of $M T D H$ expression in cell lines derived from brain obtained in HPA.

Finally, we assessed whether the expression of $M T D H$ increases with the progression of the disease. For this purpose, we used prostate cancer malignant transformation model, cell lines derived from PC3 (PC3M, PC3MLN4 and PC3MPro4). The highest level of metastatic capabilities in a mouse model of human prostate cancer reflects PC3MLN4. We found that this cell line showed the highest level of $M T D H$ expression. We confirmed that metastatic capabilities of prostate cancer cell lines correlated with an increase of $M T D H$ expression.

High expression of MTDH was also detected in the tissues and other cancer prostate cell lines. Kikuno [18] showed MTDH overexpression in clinical PC tissue samples and cultured PC cells compared to benign prostatic hyperplasia tissue samples and normal prostate epithelial cells. They proposed MTDH as a novel genetic biomarker to serve as an attractive molecular target for new anticancer agents to prevent PC cell progression and metastasis. High MTDH expression also was observed in another prostate cancer cell lines: LNCaP, DU145 [18, 19].

In addition to gene expression evaluation - Mtdh protein level was measured using human prostate tissue microarray [15]. It showed that Mtdh distribution is in the cytoplasm and cell membrane and was concluded that subcellular distribution can predict Gleason grade and survival. Furthermore, Mtdh was also found in the bone metastases of prostate cancer (81.8\%) [15]. Erdem et al. [20] examined 97 prostatectomy samples and showed that MTDH coupled with the main fibroblast growth factor is an independent prognostic parameter. These results suggest that MTDH expression, both on RNA and protein level seems to be a potential prognostic factor in prostate cancer. Moreover, the possible signalling pathways in prostate cancer cell lines, in which MTDH might be involved and that can be potential therapeutic targets for antineoplastic agents, have been studied. Inhibition of the Mtdh expression proved to be one of the molecular events demonstrating an antineoplastic effect [19]. In order to implement MTDH expression as a prognostic factor, further studies with consistent results are needed - gene expression analysis using digital droplet PCR and measurement of the potential biomarker in plasma or urine collected from prostate cancer's patients - should also be taken into account.

\section{References}

1. Garraway LA, Widlund HR, Rubin MA, et al. Integrative genomic analyses identify MITF as a lineage survival oncogene amplified in malignant melanoma. Nature. 2005; 436(7047): 117-122, doi: 10.1038/nature03664, indexed in Pubmed: 16001072. 
2. Li Y, Zou L, Li Q, et al. Amplification of LAPTM4B and YWHAZ contributes to chemotherapy resistance and recurrence of breast cancer. Nat Med. 2010; 16(2): 214-218, doi: 10.1038/nm.2090, indexed in Pubmed: 20098429.

3. van 't Veer LJ, Dai H, van de Vijver MJ, et al. Gene expression profiling predicts clinical outcome of breast cancer. Nature. 2002; 415(6871) 530-536, doi: 10.1038/415530a, indexed in Pubmed: 11823860

4. Hu G, Chong RA, Yang Q, et al. MTDH activation by $8 q 22$ genomic gain promotes chemoresistance and metastasis of poor-prognosis breas cancer. Cancer Cell. 2009; 15(1): 9-20, doi: 10.1016/j.ccr.2008.11.013, indexed in Pubmed: 19111877.

5. Su Zz, Chen Y, Kang Dc, et al. Identification and cloning of human astrocyte genes displaying elevated expression after infection with HIV-1 or exposure to HIV-1 envelope glycoprotein by rapid subtraction hybridization, RaSH. Oncogene. 2002; 21(22): 3592-3602, doi: 10.1038/sj.onc.1205445, indexed in Pubmed: 12032861.

6. Su Zz, Chen Y, Kang Dc, et al. Customized rapid subtraction hybridization $(\mathrm{RaSH})$ gene microarrays identify overlapping expression changes in human fetal astrocytes resulting from human immunodeficiency virus-1 infection or tumor necrosis factor-alpha treatment. Gene. 2003; 306: 67-78, indexed in Pubmed: 12657468.

7. Yoo BK, Emdad L, Lee SG, et al. Astrocyte elevated gene-1 (AEG-1) A multifunctional regulator of normal and abnormal physiology. Pharmacol Ther. 2011; 130(1): 1-8, doi: 10.1016/j. pharmthera.2011.01.008, indexed in Pubmed: 21256156

8. Emdad L, Sarkar D, Su ZZ, et al. Astrocyte elevated gene-1: recent insights into a novel gene involved in tumor progression, metastasis and neurodegeneration. Pharmacol Ther. 2007; 114(2): 155-170, doi 10.1016/j.pharmthera.2007.01.010, indexed in Pubmed: 17397930.

9. Meng X. Thiel KW, Leslie KK. Drug resistance mediated by AEG-1/MTDH/LYRIC. Adv Cancer Res. 2013; 120: 135-157, doi: 10.1016/B978-0-12-401676-7.00005-X, indexed in Pubmed: 23889990

10. Hu G, Wei Y, Kang Y. The multifaceted role of MTDH/AEG-1 in cancer progression. Clin Cancer Res. 2009; 15(18): 5615-5620, doi 10.1158/1078-0432.CCR-09-0049, indexed in Pubmed: 19723648

11. Zhao $Y$, Moran MS, Yang Q, et al. Metadherin regulates radioresistance in cervical cancer cells. Oncol Rep. 2012; 27(5): 1520-1526, doi: 10.3892/or.2012.1692, indexed in Pubmed: 22367022.
12. Pettaway CA, Pathak S, Greene G, et al. Selection of highly metastatic variants of different human prostatic carcinomas using orthotopic implantation in nude mice. Clin Cancer Res. 1996; 2(9): 1627-1636, indexed in Pubmed: 9816342.

13. Gnosa S, Shen YM, Wang CJ, et al. Expression of AEG-1 mRNA and protein in colorectal cancer patients and colon cancer cell lines. J Transl Med. 2012; 10: 109, doi: 10.1186/1479-5876-10-109, indexed in Pubmed: 22643064

14. Li J, Zhang Nu, Song LB, et al. Astrocyte elevated gene-1 is a novel prognostic marker for breast cancer progression and overall patient survival. Clin Cancer Res. 2008; 14(11): 3319-3326, doi: 10.1158/10780432.CCR-07-4054, indexed in Pubmed: 18519759.

15. Thirkettle HJ, Girling J, Warren AY, et al. LYRIC/AEG-1 is targeted to different subcellular compartments by ubiquitinylation and intrinsic nuclear localization signals. Clin Cancer Res. 2009; 15(9): 3003-3013, doi: 10.1158/1078-0432.CCR-08-2046, indexed in Pubmed: 19383828

16. Emdad L, Sarkar D, Lee SG, et al. Astrocyte elevated gene-1: a novel target for human glioma therapy. Mol Cancer Ther. 2010; 9(1): 79-88, doi: 10.1158/1535-7163.MCT-09-0752, indexed in Pubmed: 20053777.

17. Thul PJ, Åkesson $\mathrm{L}$, Wiking $\mathrm{M}$, et al. A subcellular map of the human proteome. Science. 2017; 356(6340), doi: 10.1126/science. aal3321, indexed in Pubmed: 28495876.

18. Kikuno $\mathrm{N}$, Shiina $\mathrm{H}$, Urakami $\mathrm{S}$, et al. Knockdown of astrocyte-elevated gene-1 inhibits prostate cancer progression through upregulation of FOXO3a activity. Oncogene. 2007; 26(55): 7647-7655, doi: 10.1038/sj.onc.1210572, indexed in Pubmed: 17563745

19. Lee HJ, Jung DB, Sohn EJ, et al. Inhibition of Hypoxia Inducible Factor Alpha and Astrocyte-Elevated Gene-1 Mediates Cryptotanshinone Exerted Antitumor Activity in Hypoxic PC-3 Cells. Evid Based Complement Alternat Med. 2012; 2012: 390957, doi: 10.1155/2012/390957, indexed in Pubmed: 23243443.

20. Erdem H, Yildirim U, Uzunlar AK, et al. Relationship among expression of basic-fibroblast growth factor, MTDH/astrocyte elevated gene-1, adenomatous polyposis coli, matrix metalloproteinase 9, and COX-2 markers with prognostic factors in prostate carcinomas. Niger J Clin Pract. 2013; 16(4): 418-423, doi: 10.4103/1119-3077.116873, indexed in Pubmed: 23974731 